\title{
GD2-specific Chimeric Antigen Receptor-modified T Cells for the treatment of Refractory and/or Recurrent Neuroblastoma in Pediatric Patients
}

Lihua Yu ( $\sim$ eveylhyu@gmail.com )

Zhujiang Hospital of Southern Medical University https://orcid.org/0000-0002-0833-3120

\section{Lulu Huang}

Zhujiang Hospital of Southern Medical University

Danna Lin

Zhujiang Hospital of Southern Medical University

Xiaorong Lai

Zhujiang Hospital of Southern Medical University

Li Wu

Zhujiang Hospital of Southern Medical University

Xu Liao

Zhujiang Hospital of Southern Medical University

Jiale Liu

Zhujiang Hospital of Southern Medical University

\section{Yinghua Zeng}

Zhujiang Hospital of Southern Medical University

\section{Lichan Liang}

Zhujiang Hospital of Southern Medical University

\section{Guanmei Zhang}

Zhujiang Hospital of Southern Medical University

\section{Bin Wang}

Zhujiang Hospital of Southern Medical University

\section{Zhu Wu}

Zhujiang Hospital of Southern Medical University

\section{Shaohua Tao}

Zhujiang Hospital of Southern Medical University

\section{Yuchen Liu}

Zhujiang Hospital of Southern Medical University

\section{Cheng Jiao}

Zhujiang Hospital of Southern Medical University

\section{Lung-Ji Chang}


Zhujiang Hospital of Southern Medical University

\section{Lihua Yang}

Zhujiang Hospital of Southern Medical University

\section{Research Article}

Keywords: neuroblastoma, CAR T, immunotherapy, pediatric, GD2

Posted Date: August 18th, 2021

DOl: https://doi.org/10.21203/rs.3.rs-803629/v1

License: (c) (1) This work is licensed under a Creative Commons Attribution 4.0 International License. Read Full License 


\title{
GD2-specific Chimeric Antigen Receptor-modified T Cells for the treatment of
}

\section{Refractory and/or Recurrent Neuroblastoma in Pediatric Patients}

Lihua $\mathrm{Yu}^{1 \dagger}$, Lulu Huang ${ }^{1 \dagger}$, Danna Lin ${ }^{1}$, Xiaorong Lai ${ }^{1}, \mathrm{Li} \mathrm{Wu}^{1}, \mathrm{Xu} \mathrm{Liao}^{1}$, Jiale Liu ${ }^{1}$, Yinghua Zeng ${ }^{1}$, Lichan Liang ${ }^{1}$, Guanmei Zhang ${ }^{1}$ Bin Wang ${ }^{1}$, Zhu Wu ${ }^{1}$, Shaohua Tao ${ }^{1}$, Yuchen Liu ${ }^{2}$, Cheng Jiao ${ }^{2}$, Lung-Ji Chang ${ }^{2 *}$, Lihua Yang ${ }^{*}$

${ }^{1}$ Department of Pediatric Hematology, Zhujiang Hospital of Southern Medical University, Guangzhou, Guangdong, China;

${ }^{2}$ Shenzhen Geno-immune Medical Institute, Shenzhen, China;

${ }^{\dagger}$ Lihua Yu and Lulu Huang contributed equally to this work.

\section{*Correspondence}

$$
\text { Lihua Yang, }
$$$$
\text { dryanglihua@163.com. ORCID ID: 0000-0002-0009-871X }
$$

Lung-Ji Chang,

c@szgimi.org

\section{Keywords neuroblastoma, CAR T, immunotherapy, pediatric, GD2}

\begin{abstract}
Purpose: This study aimed to evaluate the safety and efficacy of the fourth-generation chimeric antigen receptor (CAR) disialoganglioside 2 (GD2)-specific (4SCAR-GD2) T cells for treatment of refractory and/or recurrent neuroblastoma (NB) in pediatric patients.
\end{abstract}

Experimental Design: A phase I clinical study using 4SCAR-GD2 T cells for the treatment of NB in pediatric patients was conducted. This study was registered at www.clinicaltrials.gov (NCT02765243). A lentiviral CAR with the signaling domains of CD28/4-1BB /CD3 $\zeta-i C a s p 9$ was transduced into activated T-cells. The response to 4SCAR-GD2 T cell treatment, and 4SCARGD2 $\mathrm{T}$ cell expansion and persistence in patients were evaluated. Toxicities were determined based on the National Cancer Institute Common Terminology Criteria for Adverse Events (CTCAE) v4.03.

Results: Twelve patients were enrolled and finally ten ptients were included in this clinical trial which occurred from January 1 , 2016, to August 1, 2017. These patients had progressive disease (PD) before CAR T cell infusion. After 4SCAR-GD2 T cell treatment, $6(6 / 10)$ had stable disease (SD) at 6 months, and 4(4/10) remained SD at 1 year and alive after 3-4 years of follow up. Six patients died due to disease progression by the end of July 1, 2020. The median overall survival (OS) time was 25 months (95\% CI, 0.00 to 59.43), and the median progression-free survival (PFS) time was 8 months (95\% CI, 0.25 to 15.75). Grade 3 or 4 hematological toxicities were the common adverse events frequently occurred after fludarabine and cyclophosphamide (Flu/cy) chemotherapy. Grade 1-2 toxicities like cytokine release syndrome and neuropathic pain were common, transient and mild.

Conclusions: The 4SCAR-GD2 T cell therapy demonstrated antitumor effect and manageable toxicities, indicating its potential to benefit children with refractory and/or recurrent NB.

\section{Funding}




\section{Conflict of Interest}

The authors declare that they have no potential conflicts of interest.

\section{Data availability}

Available upon request.

\section{Author contributions}

Conception and design: Lihua Yu, Lulu Huang,Danna Lin, Yuchen Liu, LungJi Chang, and Lihua Yang. CAR T -cell production: Yuchen Liu, Cheng Jiao and LungJi Chang. Administrative, technical: Lihua Yu, Lichan Liang, Guanmei Zhang, Bin Wang, Zhu Wu, and Shaohua Tao. Acquisition, analysis, or interpretation of data: Lulu Huang, Xiaorong Lai, Li Wu, Qiulei Hu, Lichan Liang, Yinghua Zeng, and Guanmei Zhang. Drafting of the manuscript: Lihua Yu, Danna Lin, Jiale Liu and Lihua Yang. Writing, review, and/or revision of the manuscript: All authors.

Lihua Yu and Lulu Huang contributed equally to this work.

\section{Ethical Statement:}

The authors are accountable for all aspects of the work in ensuring that questions related to the accuracy or integrity of any part of the work are appropriately investigated and resolved.

\section{Informed consent:}

All participants and/or their legal guardians provided written informed consent upon enrollment and approved of the dates to further publication. 


\section{Introduction}

Neuroblastoma(NB) is the most prevalent extracranial pediatric solid tumor occurring typically in young children[1]. Patients with refractory and/or recurrent NB have a poor prognosis, even after intensive treatment with chemotherapy, surgery, stem cell transplantation, radiation, and/or antibody-based therapy[2]. Although metaiodobenzylguanidine (MIBG) and disialoganglioside 2 (GD2) monoclonal antibody treatment might be effective, they are difficult to access, resulting in worse prognosis of NB in China [3].

Disialoganglioside 2 (GD2) is a surface antigen expressed on most neuroectoderm-derived cells including NB, melanoma, and others. GD2 is also expressed less predominantly in normal human tissue cells, such as neurons, skin melanocytes, and peripheral sensory nerve fibers[4]. GD2 has been an effective target for NB immunotherapy and anti-GD2 monoclonal antibodies (MoAbs) based on the 3F8 and CH14.18 clones have been approved for clinical appplications [5; 6]. The frequent and severe pain resulting from antiGD2 antibody therapy is the main adverse event limiting dose escalation.

Chimeric antigen receptor (CAR) T cells, as a new therapeutic paradigm, have demonstrated remarkable antileukemic activity against $\mathrm{B}$ cell hematological malignancies[7; 8]. However, their efficacy against solid tumors has not yet been clearly illustrated. In a previous study, the first- generation GD2 CAR T cells comprised of EBV- cytotoxic T lymphocytes (CTLs) and conventionally activated T cells showed no neurological toxicity, and some patients with active NB even achieved complete response (CR) [9; 10]. A second-generation GD2-CAR with CD28/CD3 signaling domain T cells were well tolerated in patients without GD2 CAR-related toxicity, but they did not achieve objective clinical response. The thirdgeneration GD2 CAR T cells had similar antitumor effects, however, their effects were not greater than that of the first-generation GD2 CAR T cells [11]. Furthermore, another study revealed that the secondgeneration GD2 CAR T cells showed on-target off-tumor toxicity in patients with nuroblastoma[12].

The primary objective of this study was to evaluate the safety and efficacy of a fourth generation GD2 CAR T cells (4SCAR-GD2) for the treatment of refractory and/or recurrent NB. 4SCAR-GD2 used autologous $\mathrm{T}$ cells transduced with a lentiviral vector and was modified to express anti-GD2 antibody domain with a CD3-zeta domain, a 4-1BB (CD137) domain, a CD28 domain and an inducible caspase 9 domain. In the 4SCAR-GD2 design, we devised a suicide gene, inducible caspase 9, into the vector construct, without impairing the antitumor efficacy of the CAR T cells[13; 14; 15]. Such design could prevent severe adverse effects. This genetically engineered CAR-T cell technology was based on an extended CD28 costimulatory signal plus a CD27 signaling domain with an inducible caspase 9 suicide gene specific for CD19 surface antigen, and had been successfully applied in patients with relapsed or refractory B cell leukemia and achieved CR in up to $70-80 \%$ of patients[16; 17$]$.

\section{Methods}

\section{Study design and participants}

The study was approved by the Institutional Review Board of Zhujiang Hospital, affiliated with Southern Medical University (2016-EKZX-001). The study was registered at www.clinicaltrials.gov (NCT02765243). The protocol was conducted according to the principles of the Declaration of Helsinki. Written informed consents were provided by the guardians of all patients.

This was a single-cohort, phase I study of 4SCAR-GD2 T cells in children with refractory and/or recurrent NB. Patients received fludarabine and cyclophosphamide (Flu/cy) for lymphodepletion (Cy at 300 $\mathrm{mg} / \mathrm{m}^{2} /$ dose on days $-4,-3$, and -2 and Flu at $25 \mathrm{mg} / \mathrm{m}^{2} /$ dose on days $-4,-3$, and -2 prior to 4SCAR-GD2 T cell administration). The participants received an intravenous infusion of modified 4SCAR-GD2 T cells and were closely followed-up for treatment-related responses. The dose of infused CAR T-cells ranged from $0.13-34 \times 10^{6} / \mathrm{kg}$ body weight. Additional treatment was not administrated after 4SCAR-GD2 T cell treatment in patients without progression. 
The response to 4SCAR-GD2 T cell infusion was evaluated by computed tomography (CT), positron emission tomography (PET) or magnetic resonance imaging (MRI) on the primary tumor sites, and a bone scan and bone marrow aspirate and biopsy were performed to evaluate metastatic sites after the initial infusion, according to the international neuroblastoma response criteria (INRC) [18]. Further imaging was conducted again at 6 and 12 months after infusion. The response assessment results were recorded at 6 and 12 months after infusion. Overall survival (OS) and progression-free survival PFS were applied for evaluating the clinical response to 4SCAR-GD2 cells treatment because ${ }^{123}$ I-MIBG scans are not easily available in China. Efficacy parameters included: stable disease (SD), partial response (PR) or progressive disease (PD); complete remission (CR); PFS (time from first treatment to disease progression) and OS. Toxicities were assessed through physical examinations, performance tests and laboratory tests of organ function weekly in the first month, each month in 6 months and at the end of the study. Adverse events were graded based on the National Cancer Institute (NCI) Common Terminology Criteria for Adverse Events (CTCAE) v4.03(https://evs.nci.nih.gov/ftp1/CTCAE/CTCAE_4.03_2010-06-14_QuickReference_5x7.pdf), and cytokine release syndrome(CRS) was graded according to a revised grading system[19].

\section{GD2 expression by immunohistochemical (IHC) staining}

Formalin-fixed paraffin-embedded tumor sections were immunohistochemically stained with mouse anti-human GD2 antibody (BD Biosciences, Cat\# 554272, $0.5 \mathrm{mg} / \mathrm{mL}$ ). The isotype control was stained with purified mouse IgG2a (BioLegend, Cat\# 400202). All images were captured from tumor sections using a Zeiss Axio Vert.A1 microscope and Zeiss ZEN imaging software. The tissue staining intensity was compared with that from positive and negative controls and scored from 0 to 4 according to two components: staining intensity and the percentage of positive cells. Each sample was assessed and graded by two independent observers.

\section{Construction of the 4SCAR-GD2 lentiviral vector}

Lentiviral vectors were generated using by the NHP/TYF lentiviral vector (LV) system as previously described[20; 21]. The DNA sequences of the anti-GD2 CAR clone hu3F8 were optimized for human codons, chemically synthesized, cloned into the lentiviral vector PTYF and packaged into lentiviral particles for gene transfer[22]. The final lentiviral constructs were verified by restriction enzyme mapping and DNA sequencing. The GD2 CAR sequences were constructed with a 4th generation lentiviral CAR design (4SCAR). 4SCAR incorporated several intracellular T cells signaling motifs including the CD28 transmembrane and cytoplasmic domains, the costimulatory 4-1BB intracellular TRAF-binding domain, the $\mathrm{CD} 3 \zeta$ chain intracellular domain, and an inducible cell death-initiating caspase 9 genetic cassette (4SCARGD2) (Figure 1). The iCasp9 system has been published in previous studies, and the small molecule dimerizer drug AP1903 could induce CAR T cells apoptosis [23] .

\section{$T$ cell isolation and 4SCAR-GD2 $T$ cell production}

Peripheral blood mononuclear cells (PBMCs) of patients were collected by apheresis and blood buffy coats were prepared. PBMCs were isolated using Ficoll-Paque plus (GE Healthcare). T cells were activated using anti-CD3(MACS CD3 MicroBeads 130-097-043) and anti-CD28(Invitrogen anti-Hu CD28 clone 28.2 16-0289-85) antibody-conjugated magnetic beads. T cells were maintained in AIM-V (Invitrogen, Thermo Fisher Scientific, Waltham, MA) supplemented with interleukin IL-2, IL-7 and IL-15, as previously described[24]. Phenotype analysis of the activated cells was performed to confirm T cell purity. After expansion for two-days, T cells were transduced with the lentiviral 4SCAR-GD2 vector and quality control/assurance assay for CAR T killing function was routinely performed as an integrated program. 4SCAR-GD2 T-cells were collected and infused five days after transduction. Transduction efficiency is reported as the percentage of CAR DNA positive cells based on a standard single-cell clone tumor cell line with one copy of transgene per cell. Safety tests including mycoplasma detection and endotoxin analysis were conducted and passed before CAR T cell infusion.

\section{Immunophenotyping of $\mathbf{T}$ cell products}


The following anti-human antibodies were used to evaluate the phenotype of the cells: anti-hCD3 PE (MACS,130113129), anti-hCD4 Pacific Blue (BD Biosciences, 558116), anti-hCD8 PerCp-Cy5.5 (BD Biosciences, 565310), and anti-hCD8 PE-Cy7 (BD Biosciences, 557747). Data acquisition was performed using a CytoFLEX flow cytometer (Beckman).

\section{CAR detection by quantitative PCR (qPCR)}

The CAR copy number in the blood was determined by real time qPCR, based on both SYBR and TaqMan probe methods, as previously described[24; 25; 26; 27]. Genomic DNA was harvested from blood cells using a Promega Genomic DNA Purification Kit (Promega Corp, Madison, WI, USA). The qPCR data was collected using MX3000P (Stratagene, Agilent Technologies, Santa Clara, CA, USA) after 4SCARGD2 $\mathrm{T}$ cell treatment at $0,1,2$, and 3 weeks and each month until the CAR copy number was at undetected level.

\section{Cytokine analysis based on a cytokine bead array (CBA)}

The serum levels of the cytokines and cytokine receptors TNF- $\alpha$, IL-2R, IL-1 $\beta$, IL-8, IL- 6 and IL-10 were determined by using the BD CBA Human Soluble Protein Flex Set System at 0,1,2,3 weeks and so on[28]. The CBA system captures soluble analytes with beads of a known size and fluorescence and allows quantification using flow cytometry according to the manufacturer's manual.

\section{Statistical analysis}

Descriptive statistics (means, ranges and standard deviations or standard errors) were used to summarize data. Correlation analysis was performed using Spearman's rank correlation coefficient. OS and PFS were estimated by the Kaplan-Meier method. A two-sided P value $<0.05$ was considered statistically significant. Analyses were performed with GraphPad Prism 6 (GraphPad Software) and IBM SPSS Statistics for Windows, version 21.0 (IBM Corp., Armonk, NY, USA).

\section{Results \\ Patient characteristics}

From January 1, 2016, to August 1 2017, a total of 12 patients were screened, and 10 patients with relapsed (2/10) or refractory (8/10) disease were enrolled in the study (Figure 2). Seven of the 10 patients were male with a median age of 4.5 years (range, $1.8-7$ years). The previous treatments of the patients were chemotherapy, surgery, radiation and/or stem cell transplantation (Table S1 in the Supplementary Appendix). All patients were treated with 4SCAR-GD2 T cells (Table 1). Three patients had unresectable tumors at the primary site ( 1 in the spinal canal and 2 in the abdomen), three patients had bone marrow diseases, and the remaining three patients had multiple bone lesions, one of whom had lymph node involvement at the time of infusion. The 4SCAR-GD2 T cells could not be detected in the blood of patients No.1,2, and 7, and the guardians/families requested two or more infusions. As a result, patient No.1 received 4SCAR-GD2 T cells for three times at an interval of 3 months, while patients No.2 and 7 received 4SCARGD2 T cells for two times at intervals of 6 months and 3 months, respectively.

\section{Clinical response and survival assessments}

Patient No.1 and 7 had NB bone marrow relapse, and CAR T cells expanded poorly after infusion of 4SCAR T cells. Their 4SCAR-GD2 T cell counts in the peripheral blood were lower than detection sensitivity. Therefore, they received multiple infusions but developed bone marrow relapse at the end.

Patients No.2, 3, 4, and 9 had multiple skeletal metastatic lesions but negative in bone marrow. The efficacy of 4SCAR T cells treatment was assessed by observing survival because ${ }^{123}$ I-MIBG scans evaluation was not available. Intracranial metastases occurred in both patients No.3 and 9. Patient No.3 had a posterior cranial fossa mass and received total surgical resection, but developed skeletal recurrence 1.5 years later. Patient No.9 had intracranial metastases combined with intratumoral hemorrhage and died of brain herniation. Patients No.2 and 4 were long-term survivors. 

significant abnormality in the radiological distribution of the residual tumor was seen on PET-CT. Patient No.8 had a spinal cord occupancy that could only be partially resected by surgery, and the residual tumor was slightly smaller than before after 4SCAR-GD2 T cells therapy. Both patients were long-term survivors.

Patient No.5 and 10 had NB soft tissue residuals and developed distal site recurrence after a period of remission after 4SCAR-GD2 $\mathrm{T}$ treatment.

The clinical responses to 4SCAR-GD2 $\mathrm{T}$ cell therapy are summarized in Table 2. Of the 10 patients who had PD before CAR T cell infusion, 6 achieved SD at 6 months, and the remaining 4 patients had SD at 1 year and alive at least 3-4 years of follow-up. The median overall survival (OS) time was 25 months (95\% CI, 0.00 to 59.43), and the median progression-free (PFS) survival time was 8 months (95\% CI, 0.25 to 15.75). Six patients who relapsed again after 4SCAR-GD2 T cell infusion received salvage chemotherapy, but these patients died due to disease progression. The survival curves for all patients are shown in Figure 3.

\section{Characterization, expansion, and persistence of 4SCAR-GD2 T cells}

The median lentiviral vector gene transfer efficiency was 36.53\%, ranging from 3.59\% to 596.08\% (500\% means 5 copies of lentiviral vector genes inserted in one cell). Cell products contained both $\mathrm{CD}^{+}{ }^{+}$and $\mathrm{CD} 8{ }^{+} \mathrm{T}$ cells (median ratio CD4:CD8 $=0.81$; range, 0.15 to 4.27 ) (Table S2 in the Supplementary Appendix).4SCAR-GD2 T cells were released for infusion after a median preparation time of 7-8 days. CAR DNA copies were measured to be $0.02-22.4 \%$ in the 10 patients (Figure 4). The number of 4SCARGD2 $\mathrm{T}$ cells in patient No.10 was markedly expanded when the disease re-relapsed after six months. Moreover, the 4SCAR-GD2 T cells in the patient No.8 with 1.74\% CAR DNA copies were detected in the blood for more than 1 year (in the 17th month).

\section{Safety assessment}

Toxicities were mostly self-limited and resolved soon after treatment with the 4SCAR-GD2 T cells. There was no central neurotoxicity, and intervention or supportive care was not necessary (Table 3). No toxic event-related death was observed in this trial. The common treatment-related toxicities after Flu/cy chemotherapy conditioning were hematological events (neutropenia and thrombocytopenia) (Table S3 in the Supplementary Appendix). These events were fully reversible without any treatment in all patients within a median of 4 days (range, 0 to 22 days). Acute capillary leak syndrome (4/10) and CRS (9/10) of grades 1-2 were observed approximately 1 week after infusion. A febrile reaction (9/10) was the first and most common symptom observed. Fever without infection and febrile neutropenia occurred within a median of 10 days after infusion (range 0 to 18 days) and lasted for a mean of 5.9 days (range 0-9 days). The highest temperature was $40^{\circ} \mathrm{C}$ and lasted more than 24 hours in four patients after 4SCAR-GD2 T cell infusion at the end of the first week. Hypotension (1/10) and neuropathic pain (3/10) were rare,transient, and mild in the 4SCAR-GD2 T cell-treated patients. Cough (6/10) and acneiform rash (4/10) were common symptoms (median, 7 days; range 5 to 20 days). Eosinophilia was observed in all patients in the phase of CAR T cell expansion. There were no late-onset toxicities or long-term adverse events in the 4SCAR-GD2 T cell-treated patients during follow-up.

The patient No.10 experienced extensive papules on the face, scalp, and extremities, that were accompanied with symptoms of pruritus and tenderness with deflorescence over one month. The symptoms recovered without any treatment ((Fig. S1 in the Supplementary Appendix). Pathological biopsy suggested immune-mediated reaction.

The cytokine profiles after 4SCAR-GD2 T cell infusions were similar in all cases, with high secretion of IL-2R, IL-10, TNF- $\alpha$, IL-8, IL-6 and IL-1 $\beta$ (Figure 5). The serum levels of the cytokines were elevated when fever developed. The highest level of Ferritin, IL-6 and IL-10 were $3417 \mathrm{ug} / \mathrm{L}, 1000 \mathrm{pg} / \mathrm{ml}$ and $8014 \mathrm{pg} / \mathrm{ml}$, respectively (Table S3). There were no correlations between CAR T cell expansion and serum cytokine levels, except for the level of IL-2R $(r=0.857 ; p=0.014)$ (Fig. S2). 


\section{Discussion}

In China, patients with refractory and/or recurrent NB have a poor prognosis in the late stages[29]. This study evaluated the fourth generation GD2 CAR T-cell therapy, which was based on the CD28-4-1BB T-cell costimulatory signals plus an inducible caspase 9 domain in a lentiviral vector system. The results showed that 4SCAR-GD2 T cells were safe in children with relapsed/refractory NB. Previous studies were based on the first-, second-, and third-generation CAR T cells with both CD28 and OX40 costimulatory domains[9; $10 ; 11]$. According to preclinical and clinical studies, T-cell persistence is one of the major contributing factors for the antitumor efficacy of GD2 CAR T-cell therapies[30; 31]. CARs based on a CD28 and OX40 signaling design enhance CAR T cell survival and improve CAR T cell antitumor efficacy and clinical outcomes[32; 33]. Others studies have indicated that a $3^{\text {rd }}$-generation CAR incorporating with the CD28 and 4-1BB costimulatory domains can improve T cell persistence [34]. Therefore, the engineering of CAR T cells with a combination of multiple signaling domains has been utilized to improve the safety and efficacy of CAR T cells, and in vivo persistence [34; 35]. Because a murine antibody-based GD2-specific singlechain variable fragment ( $\mathrm{scFv}$ ) antibody could lead to $\mathrm{T}$ cell exhaustion and limit antitumor efficacy, we incorporated a humanized GD2 scFv antibody moiety in the 4SCAR-GD2 T cells, which effectively suppressed tumor growth and prolonged patients' survival [36; 37].

It has been demonstrated that Flu/cy lymphodepleting chemotherapy may enhance CAR T cell expansion and promote persistence[10; 11]. In this study, 4SCAR-GD2 T cell persistence was demonstrated to last longer than 6 months after infusion. Due to the limited number of patients, the correlation between the infusion dose and CAR T cell expansion was not found, and there was no apparent correlation between CAR $\mathrm{T}$ cell expansion and patient response. But there was a correlation between early disease progression after CAR T cell infusion and a poor prognosis after CAR T cell infusion. Furthermore, the patients with metastatic bone marrow disease and N-MYC amplification had the worst responses. The patient No. 8 who had $1.74 \%$ CAR DNA copies detected in the blood for more than 1 year, experiencd the longest survival time after the 3 years follow-up suggesting that the increased 4SCAR-GD2 T cell persistence could result in prolonged survival.

Some clinical studies support that the 4SCAR design has a high safety profile and mild cytotoxicity $[15 ; 17]$. All patients in this study didn't required the agent AP1903 to eliminate the CAR T cells because immediate severe toxicity at the time of administration and long-term toxicity were not observed.

CRS is the most common concern in CAR T cell therapy, especially CD19-specific CAR T cell therapy in patients with leukemia. Application of the anti-IL-6 receptor antibody tocilizumab is a routine management protocol[38; 39]. In this study, CRS was moderate and no severe acute adverse effects were recorded. Although positive correlations between CRS, and tumor burden or CAR T cell expansion in vivo have been reported in other CAR T cell studies[40], no such correlation was found in our study.

Toxicity of the hematopoietic system was reported after CAR-T cell therapy, especially after CD19 CAR-T cells therapy[41]. Some researchers believe that this toxicity is attributed mostly to the lymphodepleting chemotherapy regimen and CRS, characterized by a biphasic effect[42; 43]. In our study, cytopenia occurred after the lymphodepleting chemotherapy but also occurred lightly in the late period due to moderate CRS.

It is interesting that the 4SCAR-GD2 T cells markedly expanded when the disease re-relapsed (patient No.10) after six months, suggesting that re-recurrence of the tumor could stimulate the proliferation of 4SCAR-GD2 T cells again. This phenomenon supports the existence of memory 4SCAR-GD2 T cells in patients, but the rate of tumor growth might be faster than CAR T cell expansion. The high relapse rate after 4SCAR-GD2 T cell therapy remains a major challenge. Many factors contribute to a high relapse rate, such as tumor immune escape mechanisms, the immunosuppressive tumor microenvironment, perturbation of antigen presentation, and metabolic alterations in tumor cells et al[44]. 
Because no relationship between infusion dose and $\mathrm{T}$ cell expansion was observed, optimal dose of CAR T cells treatment is uncertain[8]. The tumor relapse rate might be decreased by several approaches, such as optimizing CAR density, affinity and sensing, improving immunological synapse formation, and combining treatment with oncolytic viruses or PD-1 inhibitor in the future trials[45].

In conclusion, 4SCAR-GD2 T cell therapy for relapsed and/or refractory NB patients delayed tumor progression with few toxicities, suggesting that 4SCAR-GD2 CAR T cell treatment could be a safe and efficient approach for the management of NB.

\section{Acknowledgments}

The authors would like to thank all members of the study team, the patients and their families.

\section{References}

[1] L.M. Sherief, T.H. Hassan, M. Zakaria, M. Fathy, E.A. Eshak, M.A. Bebars, and A. Esh, Tissue factor expression predicts outcome in children with neuroblastoma: A retrospective study. Oncol Lett 18 (2019) 6347-6354.

[2] S. Mallepalli, M.K. Gupta, and R. Vadde, Neuroblastoma: An updated review on biology and treatment. Curr Drug Metab (2019).

[3] Y. Su, H. Qin, C. Chen, S. Wang, S. Zhang, D. Zhang, M. Jin, Y. Peng, L. He, X. Wang, S. Qian, M. Qin, M. Ge, F. Zhang, Q. Zeng, $\mathrm{H}$. Wang, X. Ma, and X. Ni, Treatment and outcomes of 1041 pediatric patients with neuroblastoma who received multidisciplinary care in China. Pediatr Investig 4 (2020) 157-167.

[4] W.L. Ho, W.M. Hsu, M.C. Huang, K. Kadomatsu, and A. Nakagawara, Protein glycosylation in cancers and its potential therapeutic applications in neuroblastoma. J Hematol Oncol 9 (2016) 100.

[5] A.L. Yu, A.L. Gilman, M.F. Ozkaynak, W.B. London, S.G. Kreissman, H.X. Chen, M. Smith, B. Anderson, J.G. Villablanca, K.K. Matthay, H. Shimada, S.A. Grupp, R. Seeger, C.P. Reynolds, A. Buxton, R.A. Reisfeld, S.D. Gillies, S.L. Cohn, J.M. Maris, P.M. Sondel, and G. Children's Oncology, Anti-GD2 antibody with GM-CSF, interleukin-2, and isotretinoin for neuroblastoma. N Engl J Med 363 (2010) 1324-34.

[6] B.H. Kushner, K. Kramer, S. Modak, and N.K. Cheung, Successful multifold dose escalation of anti-GD2 monoclonal antibody $3 F 8$ in patients with neuroblastoma: a phase I study. J Clin Oncol 29 (2011) 1168-74.

[7] J.H. Park, I. Riviere, M. Gonen, X. Wang, B. Senechal, K.J. Curran, C. Sauter, Y. Wang, B. Santomasso, E. Mead, M. Roshal, P. Maslak, M. Davila, R.J. Brentjens, and M. Sadelain, Long-Term Follow-up of CD19 CAR Therapy in Acute Lymphoblastic Leukemia. N Engl J Med 378 (2018) 449-459.

[8] S.L. Maude, T.W. Laetsch, J. Buechner, S. Rives, M. Boyer, H. Bittencourt, P. Bader, M.R. Verneris, H.E. Stefanski, G.D. Myers, M. Qayed, B. De Moerloose, H. Hiramatsu, K. Schlis, K.L. Davis, P.L. Martin, E.R. Nemecek, G.A. Yanik, C. Peters, A. Baruchel, N. Boissel, F. Mechinaud, A. Balduzzi, J. Krueger, C.H. June, B.L. Levine, P. Wood, T. Taran, M. Leung, K.T. Mueller, Y. Zhang, K. Sen, D. Lebwohl, M.A. Pulsipher, and S.A. Grupp, Tisagenlecleucel in Children and Young Adults with B-Cell Lymphoblastic Leukemia. N Engl J Med 378 (2018) 439-448.

[9] M.A. Pule, B. Savoldo, G.D. Myers, C. Rossig, H.V. Russell, G. Dotti, M.H. Huls, E. Liu, A.P. Gee, Z. Mei, E. Yvon, H.L. Weiss, H. Liu, C.M. Rooney, H.E. Heslop, and M.K. Brenner, Virus-specific T cells engineered to coexpress tumor-specific receptors: persistence and antitumor activity in individuals with neuroblastoma. Nat Med 14 (2008) 1264-70.

[10] C.U. Louis, B. Savoldo, G. Dotti, M. Pule, E. Yvon, G.D. Myers, C. Rossig, H.V. Russell, O. Diouf, E. Liu, H. Liu, M.F. Wu, A.P. Gee, Z. Mei, C.M. Rooney, H.E. Heslop, and M.K. Brenner, Antitumor activity and long-term fate of chimeric antigen receptor-positive T cells in patients with neuroblastoma. Blood 118 (2011) 6050-6.

[11] A. Heczey, C.U. Louis, B. Savoldo, O. Dakhova, A. Durett, B. Grilley, H. Liu, M.F. Wu, Z. Mei, A. Gee, B. Mehta, H. Zhang, N. Mahmood, H. Tashiro, H.E. Heslop, G. Dotti, C.M. Rooney, and M.K. Brenner, CAR T Cells Administered in Combination with Lymphodepletion and PD-1 Inhibition to Patients with Neuroblastoma. Mol Ther 25 (2017) 2214-2224. 
[12] K. Straathof, B. Flutter, R. Wallace, N. Jain, T. Loka, S. Depani, G. Wright, S. Thomas, G.W. Cheung, T. Gileadi, S. Stafford, E. Kokalaki, J. Barton, C. Marriott, D. Rampling, O. Ogunbiyi, A.U. Akarca, T. Marafioti, S. Inglott, K. Gilmour, M. Al-Hajj, W. Day, K. McHugh, L. Biassoni, N. Sizer, C. Barton, D. Edwards, I. Dragoni, J. Silvester, K. Dyer, S. Traub, L. Elson, S. Brook, N. Westwood, L. Robson, A. Bedi, K. Howe, A. Barry, C. Duncan, G. Barone, M. Pule, and J. Anderson, Antitumor activity without on-target off-tumor toxicity of GD2-chimeric antigen receptor T cells in patients with neuroblastoma. Sci Transl Med 12 (2020).

[13] L.E. Budde, C. Berger, Y. Lin, J. Wang, X. Lin, S.E. Frayo, S.A. Brouns, D.M. Spencer, B.G. Till, M.C. Jensen, S.R. Riddell, and O.W. Press, Combining a CD20 chimeric antigen receptor and an inducible caspase 9 suicide switch to improve the efficacy and safety of T cell adoptive immunotherapy for lymphoma. PLoS One 8 (2013) e82742.

[14] T. Gargett, and M.P. Brown, The inducible caspase-9 suicide gene system as a "safety switch" to limit on-target, off-tumor toxicities of chimeric antigen receptor T cells. Front Pharmacol 5 (2014) 235.

[15] X. Xu, W. Zhao, Z. Yue, M. Qin, M. Jin, L.J. Chang, and X. Ma, 4SCAR-GD2-modified T-cell therapy in neuroblastoma with MYCN amplification: A case report with over 4-year follow-up data. Pediatr Investig 4 (2020) 55-58.

[16] J.P. Zhang, R. Zhang, S.T. Tsao, Y.C. Liu, X. Chen, D.P. Lu, P. Castillo, and L.J. Chang, Sequential allogeneic and autologous CAR-T-cell therapy to treat an immune-compromised leukemic patient. Blood Adv 2 (2018) 1691-1695.

[17] S. Tu, R. Huang, Z. Guo, L. Deng, C. Song, X. Zhou, C. Yue, L. Zhang, Y. He, J. Yang, Z. Liang, J. Du, P. Cao, Y. Li, L.J. Chang, and $\mathrm{Y}$. Li, Shortening the ex vivo culture of CD19-specific CAR T-cells retains potent efficacy against acute lymphoblastic leukemia without CAR T-cell-related encephalopathy syndrome or severe cytokine release syndrome. Am J Hematol 94 (2019) E322-E325.

[18] J.R. Park, R. Bagatell, S.L. Cohn, A.D. Pearson, J.G. Villablanca, F. Berthold, S. Burchill, A. Boubaker, K. McHugh, J.G. Nuchtern, W.B. London, N.L. Seibel, O.W. Lindwasser, J.M. Maris, P. Brock, G. Schleiermacher, R. Ladenstein, K.K. Matthay, and D. Valteau-Couanet, Revisions to the International Neuroblastoma Response Criteria: A Consensus Statement From the National Cancer Institute Clinical Trials Planning Meeting. J Clin Oncol 35 (2017) 2580-2587.

[19] D.W. Lee, R. Gardner, D.L. Porter, C.U. Louis, N. Ahmed, M. Jensen, S.A. Grupp, and C.L. Mackall, Current concepts in the diagnosis and management of cytokine release syndrome. Blood 124 (2014) 188-95.

[20] L.J. Chang, X. Liu, and J. He, Lentiviral siRNAs targeting multiple highly conserved RNA sequences of human immunodeficiency virus type 1. Gene Ther 12 (2005) 1133-44.

[21] B. Wang, J. He, C. Liu, and L.J. Chang, An effective cancer vaccine modality: lentiviral modification of dendritic cells expressing multiple cancer-specific antigens. Vaccine 24 (2006) 3477-89.

[22] M. Ahmed, J. Hu, and N.K. Cheung, Structure Based Refinement of a Humanized Monoclonal Antibody That Targets Tumor Antigen Disialoganglioside GD2. Front Immunol 5 (2014) 372.

[23] T. Gargett, and M.P. Brown, The inducible caspase-9 suicide gene system as a "safety switch" to limit on-target, off-tumor toxicities of chimeric antigen receptor T cells. Frontiers in pharmacology 5 (2014) 235.

[24] S. Okada, S. Han, E.S. Patel, L.J. Yang, and L.J. Chang, STAT3 signaling contributes to the high effector activities of interleukin-15-derived dendritic cells. Immunol Cell Biol 93 (2015) 461-71.

[25] S. Cui, C.M. Schroeder, D.Y. Zhang, and J. Meng, Rapid sample preparation method for PCR-based detection of Escherichia coli O157:H7 in ground beef. J Appl Microbiol 95 (2003) 129-34.

[26] S. Nair, V. Patel, T. Hickey, C. Maguire, D.R. Greig, W. Lee, G. Godbole, K. Grant, and M.A. Chattaway, Real-Time PCR Assay for Differentiation of Typhoidal and Nontyphoidal Salmonella. J Clin Microbiol 57 (2019).

[27] C. Zhang, X. Zheng, C. Zhao, Y. Li, S. Chen, G. Liu, C. Wang, Q. Lv, P. Liu, Y. Zheng, D. Kong, H. Jiang, and Y. Jiang, Detection of pathogenic microorganisms from bloodstream infection specimens using TaqMan array card technology. Sci Rep 8 (2018) 12828.

[28] O. Arican, M. Aral, S. Sasmaz, and P. Ciragil, Serum levels of TNF-alpha, IFN-gamma, IL-6, IL-8, IL-12, IL-17, and IL-18 in patients with active psoriasis and correlation with disease severity. Mediators Inflamm 2005 (2005) 273-9.

[29] K. Li, K. Dong, J. Gao, W. Yao, X. Xiao, and S. Zheng, Neuroblastoma management in Chinese children. J Invest Surg 25 (2012) 86-92. 
[30] C.M. Suryadevara, R. Desai, S.H. Farber, B.D. Choi, A.M. Swartz, S.H. Shen, P.C. Gedeon, D.J. Snyder, J.E. Herndon, 2nd, P. Healy, E.A. Reap, G.E. Archer, P.E. Fecci, J.H. Sampson, and L. Sanchez-Perez, Preventing Lck Activation in CAR T Cells Confers Treg Resistance but Requires 4-1BB Signaling for Them to Persist and Treat Solid Tumors in Nonlymphodepleted Hosts. Clin Cancer Res 25 (2019) 358-368.

[31] L. Jafarzadeh, E. Masoumi, K. Fallah-Mehrjardi, H.R. Mirzaei, and J. Hadjati, Prolonged Persistence of Chimeric Antigen Receptor (CAR) T Cell in Adoptive Cancer Immunotherapy: Challenges and Ways Forward. Front Immunol 11 (2020) 702.

[32] M. Prapa, S. Caldrer, C. Spano, M. Bestagno, G. Golinelli, G. Grisendi, T. Petrachi, P. Conte, E.M. Horwitz, D. Campana, P. Paolucci, and M. Dominici, A novel anti-GD2/4-1BB chimeric antigen receptor triggers neuroblastoma cell killing. Oncotarget 6 (2015) 24884-94.

[33] M. Tesfaye, and B. Savoldo, Adoptive Cell Therapy in Treating Pediatric Solid Tumors. Curr Oncol Rep 20 (2018) 73.

[34] C. Quintarelli, D. Orlando, I. Boffa, M. Guercio, V.A. Polito, A. Petretto, C. Lavarello, M. Sinibaldi, G. Weber, F. Del Bufalo, E. Giorda, M. Scarsella, S. Petrini, D. Pagliara, F. Locatelli, B. De Angelis, and I. Caruana, Choice of costimulatory domains and of cytokines determines CAR T-cell activity in neuroblastoma. Oncoimmunology 7 (2018) e1433518.

[35] M. Tanaka, H. Tashiro, B. Omer, N. Lapteva, J. Ando, M. Ngo, B. Mehta, G. Dotti, P.R. Kinchington, A.M. Leen, C. Rossig, and C.M. Rooney, Vaccination Targeting Native Receptors to Enhance the Function and Proliferation of Chimeric Antigen Receptor (CAR)-Modified T Cells. Clin Cancer Res 23 (2017) 3499-3509.

[36] S.A. Richman, S. Nunez-Cruz, B. Moghimi, L.Z. Li, Z.T. Gershenson, Z. Mourelatos, D.M. Barrett, S.A. Grupp, and M.C. Milone, High-Affinity GD2-Specific CAR T Cells Induce Fatal Encephalitis in a Preclinical Neuroblastoma Model. Cancer Immunol Res 6 (2018) 36-46.

[37] A.H. Long, W.M. Haso, J.F. Shern, K.M. Wanhainen, M. Murgai, M. Ingaramo, J.P. Smith, A.J. Walker, M.E. Kohler, V.R. Venkateshwara, R.N. Kaplan, G.H. Patterson, T.J. Fry, R.J. Orentas, and C.L. Mackall, 4-1BB costimulation ameliorates T cell exhaustion induced by tonic signaling of chimeric antigen receptors. Nat Med 21 (2015) 581-90.

[38] A. Titov, A. Petukhov, A. Staliarova, D. Motorin, E. Bulatov, O. Shuvalov, S.M. Soond, M. Piacentini, G. Melino, A. Zaritskey, and N.A. Barlev, The biological basis and clinical symptoms of CAR-T therapy-associated toxicites. Cell Death Dis 9 (2018) 897.

[39] K.T. Mueller, E. Waldron, S.A. Grupp, J.E. Levine, T.W. Laetsch, M.A. Pulsipher, M.W. Boyer, K.J. August, J. Hamilton, R. Awasthi, A.M. Stein, D. Sickert, A. Chakraborty, B.L. Levine, C.H. June, L. Tomassian, S.S. Shah, M. Leung, T. Taran, P.A. Wood, and S.L. Maude, Clinical Pharmacology of Tisagenlecleucel in B-cell Acute Lymphoblastic Leukemia. Clin Cancer Res 24 (2018) 6175-6184.

[40] R.A. Gardner, O. Finney, C. Annesley, H. Brakke, C. Summers, K. Leger, M. Bleakley, C. Brown, S. Mgebroff, K.S. Kelly-Spratt, V. Hoglund, C. Lindgren, A.P. Oron, D. Li, S.R. Riddell, J.R. Park, and M.C. Jensen, Intent-to-treat leukemia remission by CD19 CAR T cells of defined formulation and dose in children and young adults. Blood 129 (2017) 3322-3331.

[41] S. Fried, A. Avigdor, B. Bielorai, A. Meir, M.J. Besser, J. Schachter, A. Shimoni, A. Nagler, A. Toren, and E. Jacoby, Early and late hematologic toxicity following CD19 CAR-T cells. Bone Marrow Transplant 54 (2019) 1643-1650.

[42] G.R. Nahas, K.V. Komanduri, D. Pereira, M. Goodman, A.M. Jimenez, A. Beitinjaneh, T.P. Wang, and L.J. Lekakis, Incidence and risk factors associated with a syndrome of persistent cytopenias after CAR-T cell therapy (PCTT). Leuk Lymphoma 61 (2020) 940-943.

[43] A. Schaefer, C. Saygin, J. Maakaron, T. Hoelscher, Z. Purdin, J. Robinson, M. Lamprecht, S. Penza, J.E. Brammer, and Y.A. Efebera, Cytopenias after chimeric antigen receptor T-cells (CAR-T) infusion; patterns and outcomes. Biology of Blood and Marrow Transplantation 25 (2019) S171.

[44] T. Vanichapol, S. Chutipongtanate, U. Anurathapan, and S. Hongeng, Immune Escape Mechanisms and Future Prospects for Immunotherapy in Neuroblastoma. Biomed Res Int 2018 (2018) 1812535.

[45] K. Watanabe, S. Kuramitsu, A.D. Posey, Jr., and C.H. June, Expanding the Therapeutic Window for CAR T Cell Therapy in Solid Tumors: The Knowns and Unknowns of CAR T Cell Biology. Front Immunol 9 (2018) 2486. 
Figures

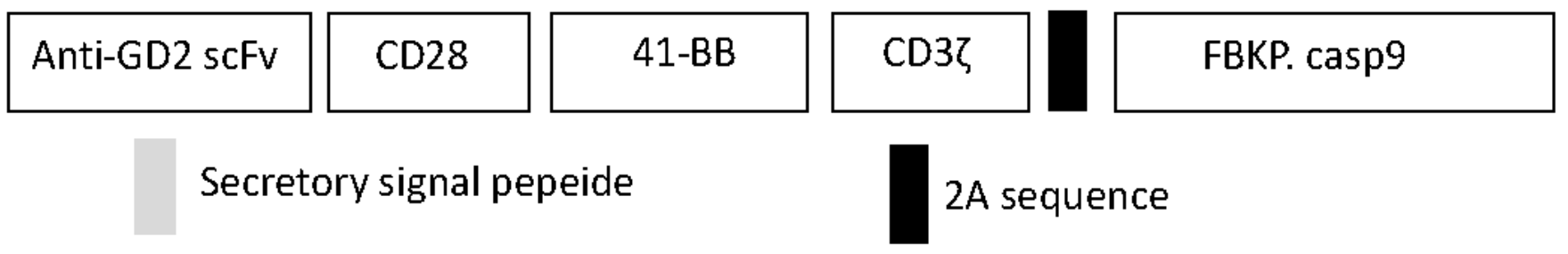

Figure 1

4SGD2-CAR T cells design: the GGGS-linker was included in all the signaling domains, and the $2 A$ linker was included in between the CD3z and iCasp9 domains

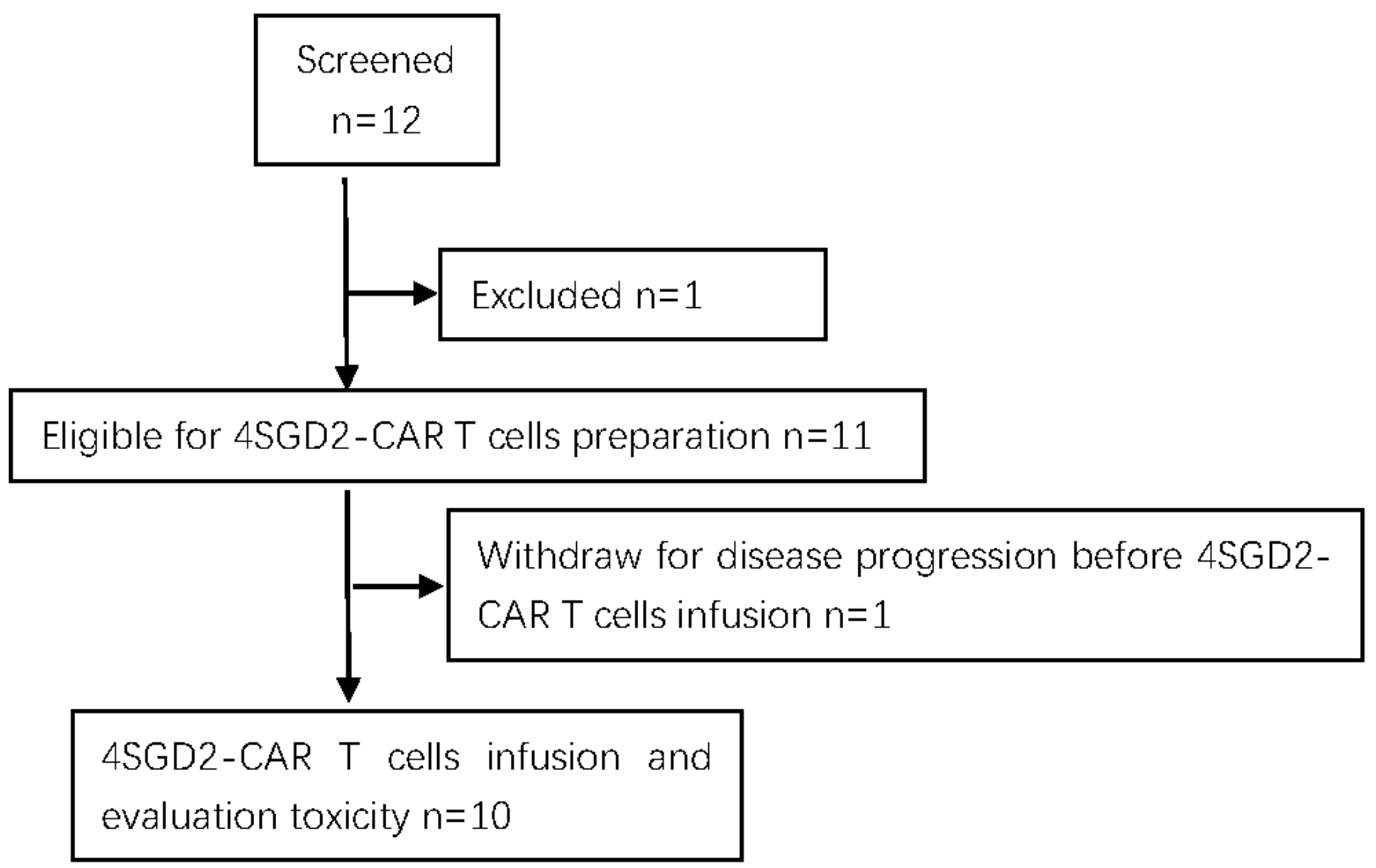

Figure 2

Recruitment and treatment on study 


\section{Progression-free and Overall Survival}

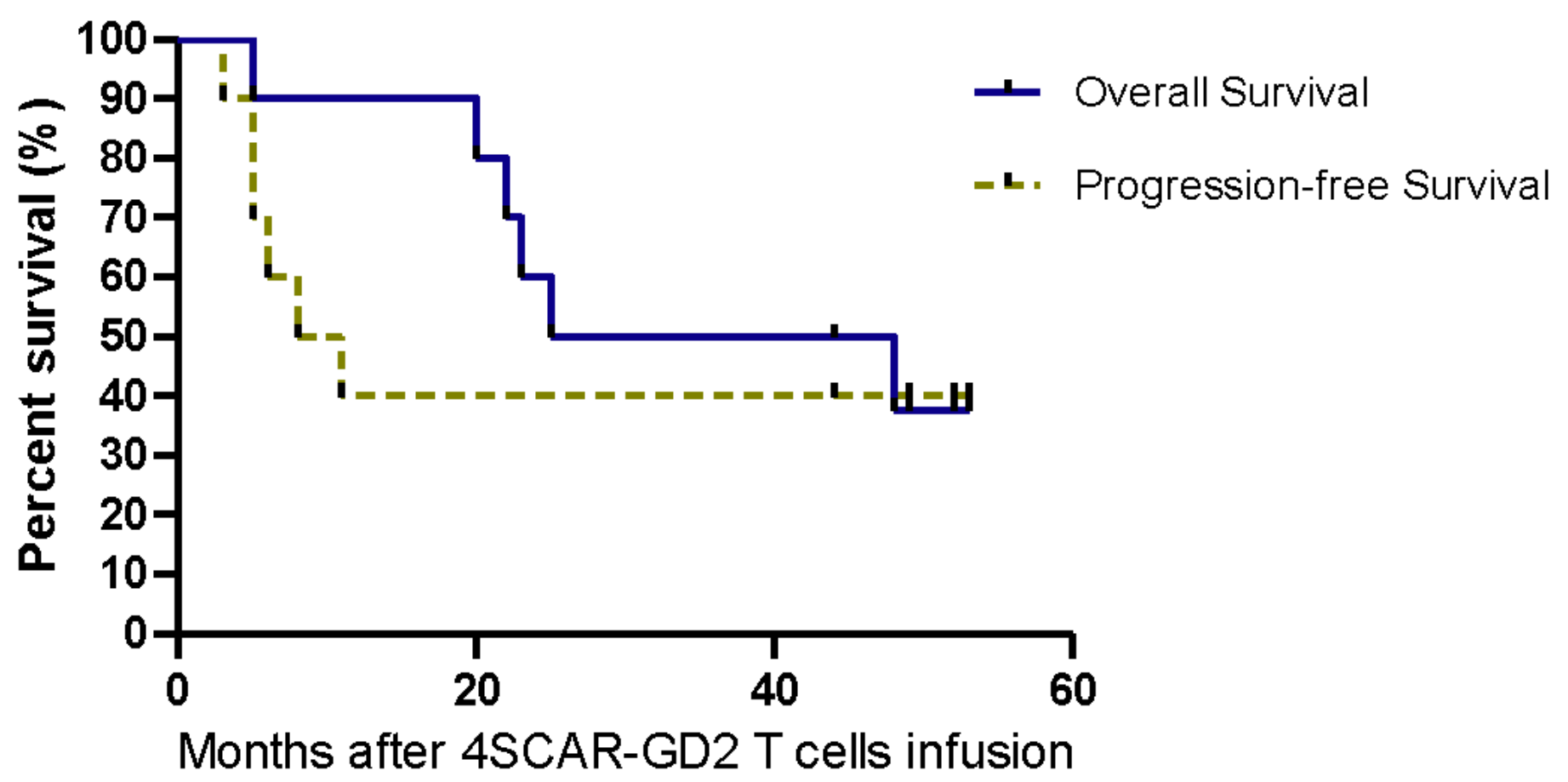

Figure 3

Long-term survival of patients who received 4SCAR-GD2 T cell therapy (PFS and OS of all the enrolled patients are shown) 


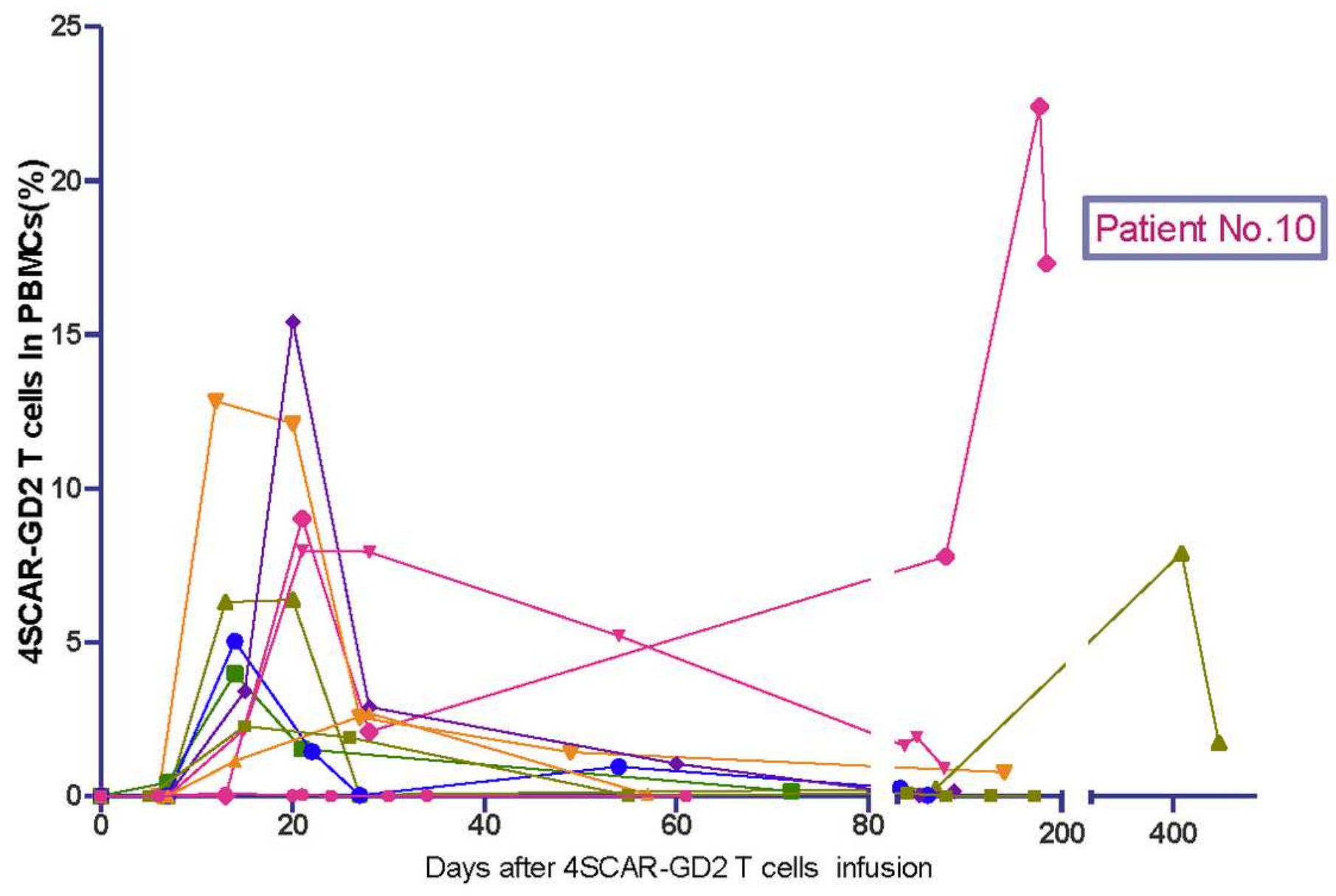

Figure 4

Kinetics of 4SCAR-GD2 T cells in the peripheral blood (Percentage of 4SCAR-GD2 T cells in peripheral blood mononuclear cells assessed by qRT-PCR before and after 4SCAR-GD2 infusion in ten enrolled patients. The 4SCAR-GD2 T cells expanded significantly when the disease re-relapsed (patient No.10) after six months 
Cytokine Levels

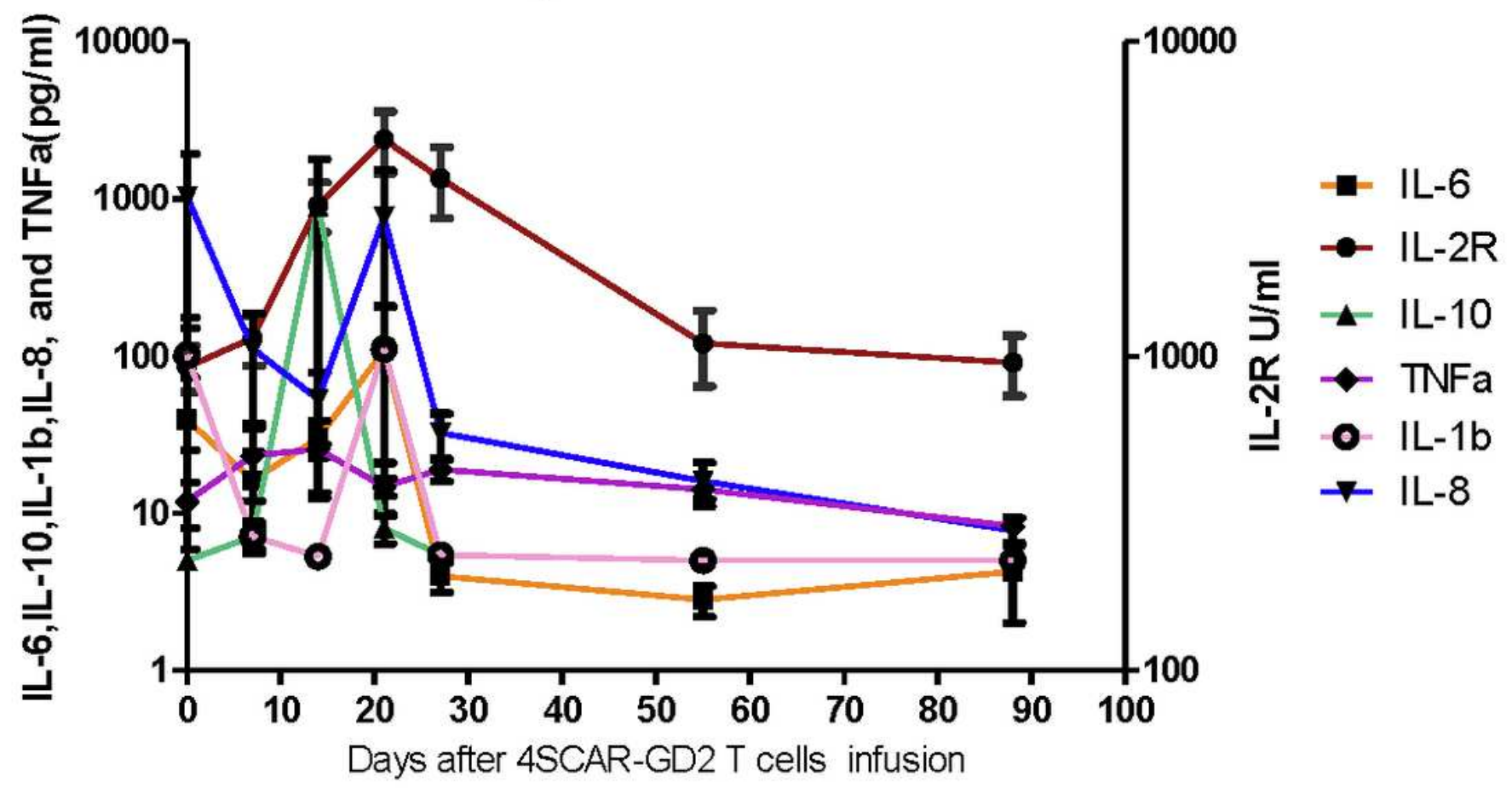

Figure 5

Serum cytokine kinetics after 4SCAR-GD2 T cell therapy (Data are presented as the Mean \pm Sem)

\section{Supplementary Files}

This is a list of supplementary files associated with this preprint. Click to download.

- TablesJOC2021811.pdf

- SupplementaryInformation2021811.pdf 